\title{
Catamenial pneumothorax sans thoracic endometrial deposits
}

\author{
R. M. T. M Gunawardena ${ }^{1}$, D. I Samaraweera ${ }^{2}$, W. Karunaratna ${ }^{3}$ \\ ${ }^{1}$ National Institute for Nephrology Dialysis and Transplantation, Maligawaththa \\ ${ }^{2}$ National Hospital of Sri Lanka. \\ ${ }^{3}$ National Hospital for Respiratory Diseases, Welisara
}

Key words: Recurrent spontaneous pneumothorax; catamenial pneumothorax; VATS pleurectomy

\section{Introduction}

Although spontaneous pneumothorax is a more common entity among males a higher number of recurrences are observed in females. Catamenial or endometrial related pneumothorax is attributed as the main cause for this high recurrence rate [1]. Recognition of this entity in a female presenting with two or more episodes of spontaneous pneumothorax is important, as treatment can prevent another episode. Here we present a case report of a 43 year old woman, who was treated for recurrent catamenial pneumothoraces at National Hospital for Respiratory Diseases (NHRD), Welisara.

\section{Case Report}

A 43 year old female was referred to NHRD following two episodes of spontaneous right sided pneumothoraces. The episodes occurred over two consecutive months, symptoms starting one day before her menstrual periods began. The first episode was treated with a tube thoracostomy at the local hospital. A contrast CT of the chest performed one day after tube thoracocostomy was unremarkable apart from a small residual pneumothorax on the right side.

She had a history of treatment for primary subfertility and a diagnostic laparoscopy done 6 years before was apparently normal. One year back she underwent excision of a lump at her left groin and the histology was that of an endometrioma.

As the clinical picture was suggestive of endometrial related recurrent spontaneous pneumothorax she was scheduled for a video assisted thoracoscopic (VATS) procedure under general anaesthesia with single lung ventilation. A three port entry was utilized. The right dome of the diaphragm was normal. The right lung was unremarkable except for a small area suspicious for scarring noted at the apex of the upper lobe. A wedge resection of that segment was done using endoGIA

Correspondence: R.M.T.M Gunawardena

E-mail: mihirangn@gmail.com

Received: 09-05-2018 Accepted: 26-07-2018

(iD) http://orcid.org/0000-0002-3867-2780

DOI: http://doi.org/10.4038/sljs.v36i3.8528

The Sri Lanka Journal of Surgery 2018; 36(3): 10-11 staplers and a parietal pleurectomy was performed. Apart from a chest infection which was treated with intravenous antibiotics her post-operative recovery was uneventful. The histology of the resected lung specimen was negative for endometrial tissue. The patient was referred back to her gynaecology team to continue their management of possible endometriosis and associated subfertility.

\section{Discussion}

Catamenial pneumothorax is defined as a spontaneous recurrent pneumothorax in a women of reproductive age, with a temporal relationship with menstruation [2]. Although the exact timing in relation to the menses is variable across the literature [2], a time frame of 24 hours before and up to 72 hours after the onset of menses is reasonable [1]. This accounts for $3-6 \%$ of spontaneous pneumothoraces in females, and the mean age of onset is between $32-35$ years [3]. The majority are seen in the right side (87.5-100\%) but rarely left sided and bilateral cases have been reported [2].

The diagnosis is entirely clinical, depending mostly on the history. The patient will present with symptoms due to pneumothorax such as chest pain, shortness of breath and cough. These symptoms are recurrent and will have a temporal relationship with menstrual periods. Primary or secondary subfertility, a diagnosis of pelvic endometriosis, a history of uterine procedures and rarely catamenial haemothorax/haemoptysis/haemopneumothorax can be associations [2]. Our patient had a history of primary subfertility. Although a diagnosis of pelvic endometriosis was not confirmed, ectopic endometrial tissue was found in the excised groin lump of hers, which fits in to the diagnosis of endometriosis.

Radiologically there are no pathognomonic features to differentiate this condition, but chest $\mathrm{X}$ ray, $\mathrm{CT}$ and rarely MRI can be helpful [2]. Elevated CA 125 levels are associated with endometriosis, and a high CA 125 level with a spontaneous pneumothorax can support a diagnosis of endometriosis associated pneumothorax [2].

The pathogenesis of this entity is still not clearly understood [4]. During menses the mucous plug occluding the opening of the uterine cervix is absent, and as a result air enters the 
peritoneal cavity through the female genital tract. This air can enter the thorax through congenital or acquired defects in the diaphragm causing a pneumothorax. Another theory postulates that prostaglandin F2 levels which increase with menstruation causes strong contractions of blood vessels and bronchioles leading to rupture of some alveoli [1, 3]. The above theories are independent of endometriosis.

Possible mechanisms for endometrial deposits in the thoracic cavity are by retrograde menstruation, via lymphatic or blood borne emboli of endometrial tissue, and metaplastic change of mesothelial cells lining the pleura [4]. During menstruation endometrial tissue deposits on the lung surface are sloughed off causing air leaks.

Characteristic pathological lesions associated with this condition are defects in the diaphragm, nodules and spots visualized in the diaphragm, visceral and parietal pleura [2]. However rarely some cases may have none of these abnormalities [2], as in our patient. In the study by Marshall et al, 2 out of $8(25 \%)$ with catamenial pneumothorax did not have any abnormality identified on initial thoracoscopy but one of them one was subsequently found to have lung parenchymal endometrial deposits when she presented with a recurrence [4]. According to Alifanao et al, out of eight patients with catamenial pneumothorax all $8(100 \%)$ had defects in the diaphragm and 7/8 (87.5\%) had histologically proven endometrial deposits in the diaphragm [5]. This strong association between diaphragmatic defects and endometrial deposits was seen in the study by Marshall et al as well and they postulated that the defects were in fact caused by the deposits [4].

Surgery is the mainstay of treatment of this condition, as it is associated with less recurrences compared to medical treatment alone with hormonal manipulation $[1,2]$. Diaphragmatic defects should be looked for and either resected or repaired [4]. Identified bullae, endometrial deposits on the lung surface should be excised/ wedge resected and a pleurodesis or a plurectomy performed [2]. A minimally invasive VATS approach is preferred $[1,2]$.
However a more invasive mini thoracotomy/thoracotomy may be necessary for reoperations or to repair a large diaphragmatic defect [2]. Surgical treatment for catamenial pneumothorax is associated with zero mortality and a negligible morbidity. However even with surgical treatment recurrence rates can be as high as $40 \%$ at 4 years [4].

Endocrine treatment should be offered for coexisting endometriosis. For those who are unfit for general anaesthesia and surgery endocrine therapy can be used as the sole treatment modality to prevent recurrences [3].

\section{Conclusions}

The diagnosis of catamenial pneumothorax as a cause for recurrent spontaneous pneumothorax in females is increasing, mainly due to heightened awareness of the condition. Once diagnosed surgery is the mainstay of treatment, preferably with a minimally invasive VATS approach. Endocrine treatment should be offered for coexisting endometriosis.

All authors disclose no conflict of interest. The study was conducted in accordance with the ethical standards of the relevant institutional or national ethics committee and the Helsinki Declaration of 1975, as revised in 2000 .

\section{References}

1. Jehangir W, Harman J, Iroka N, Yousif A. Catamenial pneumothorax: A rare cause of recurrent pneumothorax. Int J Case Rep Images 2015;6(1):51-55. doi:10.5348/ijcri-201511CR-10472.

2. Visouli AN et al. Catamenial pneumothorax. J Thorac Dis. 2014 Oct;6(Suppl 4):S448-60. doi: 10.3978.

3. Tomasz Marjański, Katarzyna Sowa, Aleksandra Czapla, Witold Rzyman. Catamenial pneumothorax - a review of the literature. doi: 10.5114/kitp.2016.61044.

4. Marshall MB, Ahmed Z, Kucharczuk JC, Kaiser LR, Shrager JB. Catamenial pneumothorax: optimal hormonal and surgical management. Eur J Cardiothorac Surg. 2005 Apr;27(4):662-6. doi: 10.1016/j.ejcts.2004.12.047.

5. Alifano M etal. Catamenial pneumothorax: a prospective study. Chest. 2003 Sep;124(3):1004-8. doi: 10.1378/chest.124.3.1004.

\section{Learning Points:}

- Catamenial pneumothorax should be considered as one cause for recurrent pneumothorax in young females.

- Endometriosis and congenital or acquired defects in the diaphragm are the most common associated factors with this condition.

- Surgery is the mainstay of treatment with a VATS approach and medical management plays a secondary role. 